\title{
BIBLIOGRAFIA
}

La presente Bibliografía, preparada por el Hispanic Institute de Columbia University para la Nueva Revista de Filología Hispánica, está en sistemática relación con la de la Revista Hispánica Moderna, en cuya Bibliografía hispanoamericana figuran los estudios referentes a Hispanoamérica.

\section{SECCION GENERAL}

\section{OBRAS BILIOGRAFICAS}

654. Bibliografía - RFH, 1945, viI, 400-416; 1946, vili, 175-193.

655. Palfrey, T. R., J. G. Fucilla \& W. C. Holbrook - A bibliographical guide to the romance languages and literatures. $33^{\mathrm{a}}$ ed. Evanston, Ill., Chandler's, 1946, Ix-84 págs., $\$ 2.75$.

656. Sierra Corella, A. - Anales bibliográficos de Madrid. (Continuación, abreviada, de la obra de Pérez Pastor). Año 1629. - BH, 1946, v, 115-124, 404-411, 484. $489,561-568,657-662,723-726$, 800-807; 1947, vi, 57-66, 150-158.

657. Vindel, Pedro - El arte ti. pográfico en España durante el siglo xv: Salamanca, Zamora, Coria y Reino de Galicia. - Madrid, Ministerio de Asuntos Exteriores, 1946, xxxi-306 págs., 400 ptas. 658. González de Amezúa, A.Cómo se hacía un libro en nuestro Siglo de Oro. - BH, 1946, v, núm. 12, 761-799.

\section{HISTORIA}

Españ $a$

659. Altamira, R. - Manual de Historia de España. 2a ed. corr. y aum. - Buenos Aires, Edit. Sudamericana, 1946, 601 págs., ilustr., $\$ 39.00 \mathrm{~m} / \mathrm{arg}$.

660. Calmette, J. - La formation de l'unité espagnole. - Paris, Flam. marion, 1946.

661. Sánchez-Albornoz, C. - El senatus visigodo: Don Rodrigo, rey legitimo de España. - CuHEsp, 1946, vi, 5-99.

662. Sánchez-Albornoz, C. - La España musulmana, según los au. tores islamitas y cristianos medievales. - Buenos Aires, Edit. "El Ateneo", s. a., 2 vols., ilustr., $\$ 48.00 \mathrm{~m} / \mathrm{arg}$.

663. SÁnchez-Albornoz, C. - Serie de documentos inéditos del reino de Asturias. - CuHEsp, 1944, I, y II, 298-351.

664. Doussinague, J. M. - Fernando el Católico y el cisma de Pisa. - Madrid, Espasa-Calpe, 1946, 706 págs.

665. González Palencia, Á.-Gonzalo Pérez, secretario de Felipe Segundo. - Madrid, Consejo Superior de Investigaciones Científicas, 1946, 2 vols.

\section{Portugal}

666. ERICeira, Conde dE - História de Portugal restaurado. Nova ed., anot. e pref. por António Ál. varo Dória. Vol. II. - Porto, Livraria Civilização, 1946, 568 págs., 50\$00. (Biblioteca Histórica. Série Régia).

667. Sanceau, Elaine - Henry the Navigator. - London, Hutchinson, 1946, 144 págs., $\$ 3.50$.

668. Diário da viagem de Vasco da 
Gama. Parte Ir. Que contém a apreciação e crítica náutica da viagem pelo Almirante Gago Coutinho e a versão portuguesa, pelo Comandante Moura Brás, do estudo exaustivo que ao Diário consagrou o Professor Franz Hümmerich. Porto, Livraria Civilização, 1946, 584 págs. $50 \$ 00$. (Biblioteca Histórica. Serie Ultramarina).

669. Ferreira, Carlos Alberto -

O sebastianismo e a aclamiação de D. João IV, segundo Fr. Manuel Homem (Subsidios histórico-bibliográficos). - Biblos, 1945, xxI, 227-241.

\section{RELIGIóN}

670. Hollis, Christopher - Saint Ignatius. - New. York, Sheed \& Ward, 1945, vir-287 págs.

671. Creixell, Juan - San Ignacio de Loyola. Ascética y Mística. Los Ejercicios Espirituales relacionados con la autobiografía del santo. Manresa, Imp. de San José, 1946, 2 vols., 45 ptas.

672. Lopes, Fernando F. - Para a história da Ordem Franciscana em Portugal: Fontes narrativas e textos legais. - AIA, 1945, v, 172-203; 1947; vIr, 31-68.

\section{CIENCIA Y ENSENANZA}

\section{España}

673. Xirau, Joaguín - La mística de Ramón Lull. - RevInd, 1945 , xxv, 323-341.

674. Xirau, Joaquín-El arte mag. no de Ramón Lull. - RNC, 1945, viI, núm. 53, 23-36; 1946, viI, núm. $54,109-133$.

675. García Blanco, M. - La casa de Nebrija en Salamanca. - Madrid, Talls. Tip. de Silverio Aguirre Torre, 1945, 24 págs. [Extr. Misceláned Nebrija, RFE, 1946, xxIx; Emérita, 1945, xIII.]

676. Clements, R. J.-The authenticity of De Hollanda's "Dialogos em Roma". - PMLA, 1946, LXI, 1018-1028.
677. Riber, L. - Erasmo y Luis Vi ves. Primera parte. BAE, 1945, xxiv, núm. 115, 193-224.

678. González, Rubén C. - Francisco de Vitoria. Estudio bibliográfico. Tesis doctoral. - Buenos Aires, Institución Cultural Española, 1946, xxvi-236 págs.

679. Homenaje a Francisco de Vitoria. - MP, 1946, xxviI, 465-522.

680. Navarro, Martín - Vida y obras de don Francisco Giner de los Ríos. - México, D. F., Ediciones Orión, 1945, 283 págs.

$$
\text { Portugal }
$$

681. Marinho, José $\div$ O pensamento filosófico de Leonardo Coimbrc. - Porto, Liv. Figueirinhas, 1945, 197 págs.

\section{ARQUEOLOGIA Y ARTE}

Espa ña

682. Morán, C. - Reseña históricoartística de la provincia de Sala. manca. - Salamanca, Universidad de Salamanca, 1946, viır-169 págs., ilustr., 30 ptas. (Acta salmanticen. sia. Filosofía y Letras, tomo II, núm. 1).

683. Lafuente (Ferrari), Enrigue -The paintings and drawings of Velázquez. Complete ed. - New York, Oxford Univ. Press, 1944, 34 págs., ilustr., \$5.50.

684. Janis, Harriet \& Sidney Janis -Picasso. The recent years: 1939. 1946. - New York; Doubleday, 1946, 211 págs., $\$ 7.50$.

\section{Portugal}

685. Macedo, Diego de - A escultura portuguesa nos séculos xvii e xviii. - Lisboa, Revista Ocidente, 1945, 135 págs., ilustr.

\section{HISPANISMO}

686. Bataillon, M. - L'hispanisme au Collège de France: Alfred Morel-Fatio. - BSS, 1947, xxiv, 132 . 139. 
687. Sánchez-Albornoz, C.--Geor ges Cirot (1870-1946). - CuHEsp, 1946, vi, 194-200.

688. Bataillon, M. - Paul Hazard et le monde ibérique. - RLComp, 1946, xx, núm. 3-4, págs. 44-50.

689. Smith, R. C. - A pioneer teacher: Father Peter Babad and his Portuguese grammar. - HispW, 1945, xxvIII, 330-363.

\section{LENGUA \\ FILOLOGIA ROMANICA}

690. Meadows, G. K. - The development of Vulgar Latin Hiatus groups in the Romance languages. - Cambridge, 1944.

691. Terracini, B. - Sobre el verbo reflexivo y el problema de los orígenes románicos. - $R F H, 1945$, viI, $1-22$.

\section{LENGUAS REGIONALES}

\section{Vasco}

692. Schuchardi, Hugo - Primitiae lingvae vasconum. Versión española con notas y comentarios de la original alemana por A. Yrigaray. Con una carta-pról. "De las dificultades de traducir al castellano algunos trabajos vascológicos de Hugo Schuchardt" por Julio de Urquijo. - Salamanca, Consejo Superior de Investigaciones Científi. cas, Colegio Trilingüe de la Universidad, 1947, 88 págs. (Tesis y Estudios Salmantinos, III).

693. Altube, S. de - Fonética y etimología euskéricas. - Gern, 1947, I, 29-33.

694. Gorostiaga, J. - La semana vasca: el sistema y los nombres de los días. - Gern, 1947, 1, 49-54.

\section{HISTORIA DEL IOMA}

Español

695. Bibliografía de Amado Alonso.

Homenaje de sus discípulos.-Buenos Aires, Coni, 1946, 46 págs.

696. Hatzfeld, H. - Hispanic phi- lology in Latin America. - Americ, 1947, III, núm. 3, 347-362.

697. Alonso, Amado - Sobre: R. Menéndez Pidal, La unidad del idioma \& Amado Alonso, La Argentina y la nivelación del idioma. - RFH, 1944, vi, 402-409.

698. Alonso, Amado - Sobre: R. Menéndez Pidal, Castilla, la tradición, el idioma. - RFH, 1945, VII, 284-288.

699. Rodríguez Demorizi, E. - Vicisitudes de la lengua española en Santo Domingo. Discurso de ingreso en la Academia Dominicana de la Lengua. Contestación del Li. cenciado Virgilio Díaz Ordóñez. - Ciudad Trujillo, R. D., Editora Montalvo, 1944, 25 págs.

\section{Portugal}

700. Boléo, Manuel de Paiva Introdução ao estudo da filologia portuguesd. - Lisboa, Ediçăo da "Revista de Portugal", 1946, virI150 págs.

701. Leño, Duarte Nunes de Origem da língua portuguesa. $4^{a}$ cd. conforme a primeira, com estudo preliminar e anotaçōes de José Pedro Machado. - Lisboa, Pro Domo, 1945, xvir-363 págs.

\section{GRAMATICA}

Español

702. Malkiel, Y. - Development of the Latin suffixes -antia und -entia in the Romance languages, with special regard to Ibero-Romance. - Berkeley and Los Angeles, University of California Press, 1945. (University of California Publications in Linguistics, vol. I, núm. 4 , 41-188.)

703. Selva, J. B. - Los sufijos en el crecimiento del habla. - BAAL, 1945, XIv, 387-458.

704. Malkiel, Y. - Old Spanish nadi(e), otri(e). - HR, 1945, xIIr, 204-230.

705. KANY, C. E. - American-Spa- 
nish syntax. - Chicago, University of Chicago Press, 1945, xII-463 págs., $\$ 6.00$.

706. Bolinger, D. L. — «Qué tan, qué tanto» - HR, 1946, xIV, 167-169.

Portugués

707. Hald, Jr., R. A.-Brazilian Portuguese inflection. - HR, 1945, XIII, 231-242.

708. Barrett, L. L. - Position of the Brazilian pronoun object of the infinitive. HR, 1945, xIII, 340-346.

\section{Enseñanza del idioma}

709. D'Eça, Raul \& E. V. GreenFIELD - An outline of Portuguese grammar. - New York, Barnes \& Noble, [1947], 199 págs., \$1.25. (College Outline Series).

710. A graded word book of Brazilian Portuguese. Compiled and edited by C. B. Brown, W. M. Carr \& M. L. Shane. Issued by the Committee on Modern Languages of the American Council on Education. - New York, F. S. Crofts, 1945, IX-252 págs., $\$ 2.00$.

\section{FONETICA}

\section{Español}

711. Cuervo, Rufino J. - Acentuación de las voces hebreas en castellano.-BICC, 1945, I, 205-211.

712. Predmore, R. L. - Notes on Spanish consonant phonemes. HR, 1946, xIV, 169-172.

713. Alonso, Amado - Nota sobre una ley fonológica del español. HR, 1947, xv, 306-307.

714. Alonso, Amado - Las correspondencias arábigo-españolas en los sistemas de sibilantes. - RFH, 1946, viri, 12-76.

715. Alonso, Amado - Árabe st> esp. ç. - esp. st $>a$ rabe ch. PMLA, 1947, LXII, 325-338.

\section{Portugués}

716. Stavrou, C. - Brazilian-Portuguese pronunciation. - New York,
David McKay, 1947, 152 págs., $\$ 2.50$.

717. Amaral, Vasco Botelho de - Estudos críticos de língua portuguesa. I. As bases da ortografia luso-brasileira. (Análise crítica. Anotaçōes filológicas. Indice alfabético e remissivo). - Lisboa, 1946, 176 págs.

\section{METRICA}

718. SaAvedra Molina, J. - Tres grandes metros: el eneasílabo, el tredecasílabo y el endecasílabo. Santiago de Chile, Prensas de la Universidad de Chile, 1946, 122 págs.

719. SaAvedra Molina, J. - El verso de arte mayor. - AUCh, 1945, cIII, núms. 57-58, 5-127.

720. Henríquez Ureña, Pedro Sobre la historia del alejandrino. RFH, 1946, vIII, 1-11.

721. BASSAGODA, R. DE - Del alejandrino al verso libre.-BAAL, 1947, xvi, 65-113.

\section{LEXICOGRAFIA}

Español

722. Cuervo, Rufino José - Diccionario de construcción y régimen de la lengua castellana. - BICC, 1946, II, 85-165.

723. Boggs, R. S., L. Kasten, H. Keniston \& H. B. Richardson Tentative dictionary of Medieval Spanish. - Chapel Hill, N. C., R. S. Boggs, 1946, 2 vols., \$3.00. 724. Tamayo, J. A. - Fortuna de un libro sobre sinónimos españoles: El "Examen" de López de la Huer. ta. - BH, 1945, Iv, 242-253.

725. Baralt, Rafael María-Diccionario de galicismos, voces, locuciones y frases. Pról. de Juan Eugenio Hartzenbusch. Primera ed. argentina con índice general de vocablos, frases y ejemplos, por Joaquín Gil. - Buenos Aires, Joaquín Gil, 1945, 832 págs., 88 ptas.

726. Serralles, J. K. - English- 
Spanish and Spanish-English dictionary of aviation terms. - New York, McGraw-Hill Book Company, 1944, x-131 págs., $\$ 2.50$.

727. González de la Calle, P. U. - Adiciones lexicológicas.-BICC, 1946, II, 12-38.

728. Selva, J. B. - Verbos nuevos. - BAAL, 1946, xv, 179-192.

729. Oliver Asín, J. - El árabe mary en el vocabulario romance $y$ en la toponimia de España. BAE, 1945, xxiv, núm. 115, 151176.

730. Pérez Vidal, J. - Fichas para un vocabulario canario. - $\mathrm{La} \mathrm{La}$ guna de Tenerife, Canarias, $\mathrm{Fa}$ cultad de Filosofía y Letras de la Universidad de la Laguna, 1945, 14 págs.

731. Pérez, Juan Régulo - Cuestionario sobre palabras y cosas de la Isla de la Palma. - La Laguna de Tenerife, 1946, 185 págs.

732. Malaret, A. - Diccionario de americanismos. Suplemento. Tomo II: F-Z. - Buenos Aires, Academia Argentina de Letras, 1944, 520 págs.

733. Malaret, A. - Diccionario de americanismos. 2ạ ed. Novísimo suplemento. - BF, 1945, IV, 136159.

734. Malaret, A. - Los americanismos a través de los siglos. UnivCB, 1945, xI, 177-194.

735. Granada, D. - Apuntamientos sobre lexicografía americana con especial aplicación al Río de la Plata; Apuntamientos para el estudio de la lexicografía americana en los países de habla española y en especial del $R$. de la Plata. BAAL, 1946, xv, 287-365.

736. Granada, D. - Sobre los nombres de los colores del caballo de América. - BAAL, 1946, xv, 671. 677.

737. Manso, M. - Las voces de Martín Fierro. - Tucumán, ed. del autor, 1945.

738. Spitzer, L. - Etimologías his- pánicas. [Artesa, artesón; barahunda; ceñiglo, vestiglo; catalán "migrarse"] - BICC, 1946, II, I-11.

739. Bourciez, I. - Hispánica: A propos du vieil espagnol "ondrar". Sur quelques emprunts aragonais au Gascon. - BHi, 1945, XLvII, 161-168.

740. Spitzer, L. - Debailadas-bailar. - BAAL, 1945, xIv, 729 . 735.

741. Consulta acerca de la palabra machaza.-BAAL, 1945, xIv, 738740 .

742. Henríquez Ureña, Pedro Sobre: Lawrence B. Kiddle, The Spanish word jicara: A word history. With an appendix on the manufacture of jícaras in Olinalá, Guerrero. - RFH, 1945, vir, 288290.

743. García Blanco, M. - Una cuestión de lexicografía medieval. Falifa, Falifo: 'prenda de vestir'. - BAE, 1946, xxv, núm. 118, 221-250.

744. Duncan, R. M. - Meaning of pimienta in medieval Spanish. - HR, 1946, xIv, 66-68.

745. Malkiel, Y. - The word family of old Spanish recudir. HR, 1946, xIv, 104-159.

746. Malkiet, Y. - Antiguo judeoaragonés, aladma, alalma 'excomunión'. - RFH, 1946, viII, 136. 141.

\section{Portugués}

747. Torrinha, F. - Novo dicionário da língua portuguêsa para os estudantes e para o povo. - Pôrto, Tip. Domingos Barreira, 1945, 1328 págs.

748. Michaelis, M. - Novo dicio nario da lingua portugueza $e$ ingle$z a$, enriquecido com os termos technicos do commercio e da industria, das sciencias $e$ das artes e da linguagem familiar. - New York, Frederick Ungar Publishing Co., s. f., 729 págs.

749. Viotti, M. - Dicionário da gíria brasileira. - São Paulo, Ed. 
Universitária, 1945, 372 págs., $\mathrm{Cr}$ 40\$00. (Bibl. Cultura Geral).

750. Fonseca, Fernando Venâncio Peixoto DA - As palavras inglesas de origem portuguesa ou importadas através do português. RFL, 1946, xII, núms. 1-2, 74-86.

751. JABERG, K. - Géographie linguistique et expressivisme phonétique: Les noms de la balançoire en portugais. - Coimbra, 1946.

752. Merêa, P. - Vária: Dois problemas filológico-jurídicos: I. Manquadra. II. Filho de gança. - Biblos, 1945, xxi, 243-249.

\section{DIALECTOLOGFA}

\section{Peninsular}

España

753. García de Diego, V. - Manual de dialectología española. Madrid, Instituto de Cultura Hispánica, 1946, 324 págs., 30 ptas.

754. Rosenblat, A.-Notas de morfología dialectal. - Buenos Aires, Instituto de Filología, 1946. (Biblioteca de Dialectología Hispanoamericana, II, 103-394).

755. Spitzer, L. - Salmantino en íteles y véntiles. - RFH, 1946, virI, $130-132$.

\section{Portugal}

756. Teixeira, J. A. - Estudos de dialectologia portuguesa. Linguagem de Goiás. Vol. II. - São Paulo, Anchieta, 1944, 169 págs., Cr $15 \$ 00$.

\section{Extra-Peninsular}

757. Espinosa, A. M. - Estudios sobre el español de Nuevo Méjico. Parte Ir: Morfología. Trad., reelaboración y notas de Ángel Rosenblat. - Buenos Aires, Instituto de Filología, 1946. (Biblioteca de Dialectología Hispanoamericana, II, págs. 1-102.)

758. Kany, C. E. - Some aspects of Bolivian popular speech. - HR, 1947, xv, 193-205.
759. WÁshIngton Bermúdez; S. El lunfardo y el lenguaje vernáculo. - BF, 1945, Iv, 180-184.

760. Bujaldón, A. R. - El adverbio allú. - AILing, 1942, II, 172-175.

\section{LITERATURA \\ LITERATURA GENERAL}

761. Columbia dictionary of modern European literature. Horatio Smith, general editor. - New York; Columbia University Press, 1947, xIv899 págs., $\$ 10.00$.

\section{LITERATURA HISPANOARABE}

762. Asín Palacios, Miguel-Obras escogidas. I. - Madrid, Consejo Superior de Investigaciones Científicas, 1946, 336 págs.

\section{LITERATURA HISPANOJUDAICA}

\section{Edad Media}

763. KAHN, M. J. - Los poetas judeoespañoles de la plegaria. UNC, 1946, núm. 7, 53-67.

764. Poesías de Yehudá Haleví. Trad. por Rosa Castillo de Moreno. Insu, 1945, II, 188-192.

\section{LITERATURAS REGIONALES}

\section{Catalana}

765. Brown, R. F. - The romantic novel in Catalonia.- $H R, 1945$, xIII, 294-323.

766. PAGÈs, A. - La chanson catalane du Navarrais Francesch de Amezcua sur l'Immaculée-Conception. - BHi, 1945, xLVII, 26-33.

767. Martínez, Pero. Obras. Ed., pról. y notas por M. de Riquer. Barcelona, Consejo Superior de Investigaciones Científicas, 1946, 152 págs.

768. Verdaguer, Jacinto-L'Atlántida. Primera ed. crítica según dos manuscritos autógrafos y las ediciones de 1877 y 1878 , preparada por E. Junyent.-Barcelona, Avun- 
tamiento, 1946, xxir-206 págs., 50 ptas.

769. Pons, J. S. - Jacint Verdaguer. - BHi, 1945, xLvII, 199-218.

770. Casanovas, D. - Vardaguer, poeta de la Atlántida. - RNC, 1945, viI, núm. 51, 75-84.

771. Díaz-Plaja, G. - En el 50 aniversario de "Tierra baja" [de Ángel Guimerá]. - BAAL, 1946, $\mathrm{xv}, 155-176$.

\section{Gallega}

772. Carnes, Luisa -- Rosalía de Castro. (Raíz apasionada de $\mathrm{Ga}$ licia.) - México, D. F., Ediciones Rex, 1945, 123 págs.

\section{HISTORIA LITERARIA}

\section{España}

773. Valbuena Prat, Á. - Historia de la literatura española. $2^{\mathrm{a}} \mathrm{ed}$., corr. y aum. - Barcelona, Gustavo Gili, 1946, 2 vols.

774. Bell, A. F. G. - The Spanish attitude towards nature. - BSS, 1947, xxIv, 74-78.

775. Menéndez Pidal, Ramón Idea imperial de Carlos V. La condesa traidora. El romanz del Infant García. Adefonsus Imperator Toletanus. - Madrid, Espasa-Calpe, 1945, 163 págs., 4.50 ptas.

776. González Palencia, Á. - Del "Lazarillo" a Quevedo. Estudios histórico-literarios. 4a serie. - Madrid, Consejo Superior de Investigaciones Científicas, 1946, viII-430 págs.

777. Green, O. H. - A critical survey of scholarship in the field of Spanish Renaissance literature: 1914-1944. - SPh, 1947, xLIv, 228-264.

778. VIAN, F.-Introduzione alla letteratura spagnola del "Siglo de Oro". - Milano, Società Editrice "Vita e Pensiero", 1946, 122 págs., L. 250 .

779. JeschKe, H. - La generación de 1898 en España. (Ensayo de una determinación de su esencia.) Trad., introd. y notas de Y. Pino Saavedra. - Santiago de Chile, Ediciones de la Universidad de Chile, 1946, 214 págs.

780. Mathews, E. G. - English translations from Spanish: A review and a contribution. - IEGPh, 1945, XLIV, 387-424.

\section{Portugal}

781. Cidade, H. - A literatura portuguesa e a expansão ultramarina. As idéias. Os sentimentos. As formas de arte. Vol. r. (Séculos $\mathrm{xv} \mathrm{e}$ xvi). - Lisboa, Agência Geral das Colónias, 1943, 293 págs.

\section{TEMAS}

782. Fernández Moreno, C. - Pelayo y los románticos. Vida literaria del fundador de la raza. Conferencia. - Buenos Aires, Publicación del Centro Asturiano de Buenos Aires, Ateneo Jovellanos, 1947, 37 págs.

\section{RELACIONES LITERARIAS}

\section{Influencias hispánicas}

783. Tinianova, I. - Analogías españolas en la obra de Gogol. Trad. de Isabel Vicente. - LitIn, 1945, Iv, núm. 8, 63-65 \& 86.

784. SAcks, Z. - Verdi and Spanish romantic drama. - HispW, 1944, xxvII, 451-465.

\section{Obras extranjeras inspiradas en temas hispánicos}

785. Hopkinson, Tom - Mist in the Tagus.-Boston, Little, Brown \& Co., 1947, 249 págs., \$2.50.

\section{Influencias extranjeras}

786. Marasso, A.-Hesíodo en la literatura castellana.-BAAL, 1947, Xvi, 7-63.

787. Marasso, A. - Píndaro en la literatura castellana.-BAAL, 1946, $\mathrm{xv}, 7-55$. 
788. Silió Cortés, C. - Maquiavelo y su tiempo. (Repercusión del maquiavelismo en las teorías y en las prácticas de gobierno.) - Madrid, Espasa-Calpe, 1946, 288 págs.

789. Lima, Henrigue de Campos Ferreira - O poeta suiço Salomão Gessner em Portugal. (Notas bibliográficas ).-Biblos, 1945, xxI, 27-36.

\section{Traducciones}

790. Cântico dos cânticos. - São Paulo, Ediçóes Cultura, 1944, 236 págs., 30\$00. (Os Mestres do Pensamento). [Traducciones y paráfrasis de João de Deus, José Beneditto Cohen, y Jamil Almansur Haddad].

791. Esquilo - Prometeo encadenado. Pról., sel. y notas de J. D. García Bacca. - México, Secretaría de Educación Pública, 1946, 74 págs. (Biblioteca Enciclopédica Popular).

792. Rodríguez-MoÑrno, A. - La edición más antigua de la "Eneida" en castellano. (Burgos, 1528). -BH, 1947, viı, núm. I, 27-34.

793. Rimbaud, Jean Arthur - Poesías. Sel., versión y pról. de V. Gaos. - Madrid, Imp. Uguina, 1946, 76 págs., 6 ptas.

794. Keats, John - Poesías. Sel., versión y pról. de Clemencia Miró. - Madrid, Edit. Hispánica, 1946, 67 págs., 6 ptas.

795. Rilke, Rainer Maria - Requiem. Las elegías de Duino. Texto original alemán, conversión castellana e introd. por G. Torrente Ballester-Madrid, Ediciones Nueva Época, 1946, 183 págs., 45 ptas.

\section{AUTORES Y OBRAS DE GENEROS DIVERSOS}

España

796. León, LUIS DE - Obra escogida. Sel., pról. y notas de Juan José Domenchina. - México, s. a., 166 págs.
797. Quevedo y Villegas, FranCISCO DE - Vida del Buscón. Sueños $y$ discursos. Textos descubiertos y clasificados por L. Astrana Marín. - Madrid, Aguilar, 1945, 505 págs., 15 ptas.

798. Luis Astrana Marín - Epistolario completo de D. Francisco de Quevedo Villegas. Ed. crítica (con extensas anotaciones, apéndices, documento inéditos y una acabada bibliografía). - Madrid, Instituto Editorial Reus, 1946, XLIX834 págs., 80 ptas.

799. Homenaje a D. Francisco Gómez de Quevedo y Villegas: 16451945. - RevInd, 1945, xxv, 403464 , ilustr.

800. Isaza Calderón, B. - Don Francisco de Quevedo y Villegas (1580-1645).-BAPaL, 1945, núm. 4, 37-56; reimpr. UnivPan, 1946, núm. $24,53-74$.

801. López de Mesa, L. - Don Francisco de Quevedo y el Renacimiento español. - RAmer, 1945 , Iv, 177-188.

802. Cossío y Corral, A. DE - Genio y figura de Don Francisco de Quevedo. - AUH, 1946, Ix, núm. 2, 5-24.

803. Amezúa, A. G. DE - Las almas de Quevedo. - BAE, 1946, Xxv, núm. 118, 251-298.

804. García Blanco, M. - Don Miguel de Unamuno y sus seudónimos. Homenaje a su memoria en el décimo aniversario de su muerte. - BSS, 1947, xxiv, 125132.

805. Farinelit, A. - Il conflitto tragico nell'anima $e$ nel pensiero di Unamuno. - BSS, 1947, xxiv, 117-125.

806. Grau, Jacinto - Unamuno, su tiempo y su España. - Buenos Aires, Edit. Alda, 1946, 196 págs.; $\$ 4.50 \mathrm{~m} /$ arg.

807. TARragó, A. - Unamuno y !a formación del "intelectual". - A, 1946, LxxxIv, 319-331. 
Portugal

808. Garret, Almeida - Poesid $e$ teatro. Ensaio preambular, sel. e notas por Mário Gonçalves Viana. - Pôrto, Livraria Figueirinhas, 1944, 207 págs.

\section{POESía}

809. SÁinz de Robles, F. C. - Historia y antología de la poesía castellana (del siglo xii al $x x$ ). Ed. ilustrada con prólogos, notas, vocabularios e índices.-Madrid, Aguilar, 1946, 1718 págs., 125 ptas.

810. Menéndez Pidal, R. - Poesía juglaresca y juglares. Aspectos de la historia literaria y cultural de España. 2a ed. - Buenos Aires, Espasa-Calpe, 1945, 280 págs.

811. Alonso, D. - Ensayos sobre poesía española. - Buenos Aires, Revista de Occidente Argentina, 1947, 401 págs.

812. Vossler, K. - La poesía de la soledad en España. - Buenos Aires, Losada, 1946, 398 págs., \$25.00 $\mathrm{m}$ /arg. (Estudios literarios).

813. Lida, María Rosa - El amanecer mitológico en la poesía narrativa española. - RFH, 1946, viII, 77-110.

814. Camarena, J. - Bibliografía de fabulistas españoles. - $\mathrm{BH}$, 1947, vi, núm. 1, 67-72; núm. 2, 159-163; núm. 3, 279-282.

815. Camp, Jean - La guirlande espagnole. Les cent plus belles fleurs du sonnet espagnol transplantées en terre française. Pról. de Alfonso Reyes. - México, D. F., Editions Le Coq Français, 1947, vir-201. págs.

816. Poesía de amor. Antologia portuguesa. Sel. e pref. de José Régio e Alberto de Serpa. - Pôrto, Livraria Tavares Martins, 1945, 302 págs.

\section{Épica}

817. Menéndez Pidal, R. - La epopeya castellana a través de la literatura española. - Buenos Ai- res, Espasa-Calpe, 1946, $\$ 9.00$ $\mathrm{m} / \mathrm{arg}$.

818. Cirot, G. - L'épisode des Infants de Carrion la rouvrarie de Corpes et le retour à Valence) dans le "Mio Cid" et la Chronique générale. - BHi, 1946, xLviri, 64-74.

819. Cirot, G.-L'affaire des malles du Cid. - BHi, 1946, xLviII, 170. 177.

820. Spitzer, L. - ¡Dios, qué buen vassallo si oviesse buen señor! RFH, 1946, vIII, 132-136.

821. Salinas, Pedro - La vuelta al esposo: ensayo sobre estructura $y$ sensibilidad en el "Cantar de Mia Cid". - BSS, 1947, xxiv, 79-88.

822. Poema de Fernán González. Ed., pról. y notas de A. Zamora Vicente. - Madrid, Espasa-Calpe, 1946. (Clásicos Castellanos).

823. Fucilla, J. G. - Bernardo de Balbuena's Siglo de Oro and its sources. - HR, 1947, xv, 101119.

824. Pierce, F. - The canto épico of the seventeenth and eighteenth centuries. - HR, 1947, xv, 1-48.

\section{Romancero}

825. Díaz y de Ovando, ClementINA - Agua, viento, fuego y tierra en el romancero español. AIIE, 1945, III, núm. 12, 59-83.

826. Spitzer, L. - Los romances españoles: El romance de Abenámar. - Asom, 1945, I, núm. 1, págs. 7-29.

\section{Autores antiguos}

$E s p a \tilde{n} a$

827. Cirot, G. - Inventaire estimatif du "mester de clerecía". BHi, 1946, xLVIII, 193-209.

828. Lida, María Rosa - Notas para el texto del Alexandre $y$ para las fuentes del Fernán González. - RFH, 1945, vir, 47-51.

829. Lida, María Rosa - La hipérbole sagrada en la poesía cas- 
tellana del siglo $x v .-\mathrm{RFH}, 1946$, viII, 121-130.

830. Avalie Arce, J. B. - Sobre Juan Alfonso de Baena. - RFH, 1946, vIII, 141-147.

831. Ribeiro, Mário de Sampayo - Uma nota ao "Cancioneiro Geral" de Garcia de Resende: As trovas "das pãcadas dos cantores" lidas por um músico.-Biblos, 1945, xxI, 1-26.

832. Carilla, E. - Restituciones a la lírica española. - RFH, 1946, virr, $148-150$.

833. Cossío, J. M. DE - Rodrigo de Reinosa y sus obras. - BBMP, 1945, xxi, núm. 1, págs. 9-70.

834. Garcilaso de la Vega - Poesías. Homenajes de Rafael Alberti. - Buenos Aires, Edit. Pleamar, 1946, 225 págs.

835. Bell, A. F. G. - Sobre: E. Allison Peers, St John of the Cross and other lectures and addresses, 1920-1945. - BSS, 1946, xxIII, 282-285.

836. García Blanco, M.-San Juan de la Cruz y el lenguaje del siglo xvi. - Valladolid, Tip. Cuesta, 1945, 21 págs. (Universidad de Valladolid. Facultad de Filosofía y Letras).

837. Peers, E. A. - El misticismo en las poesías originales de Fray Luis de León. - BBMP, 1946, XxII, 111-131.

838. Green, O. H. - Vida y obras de Lupercio Leonardo de Argensola. - Zaragoza, (Consejo Superior d e Investigaciones Científicas), 1945, 194 págs.

839. BarbazÁn, J. - Una edición ignorada de un "romance" de Lope de Vega. - BH, 1946, v, núm. 3, 157-167.

840. Lapesa, R-La "Jerusalén" del Tasso y la de Lope. - BAE, 1946, xxv, núm. 117, págs. 111-136.

841. La canción a las ruinas de Itálica del Licenciado Rodrigo Caro. Con introd, versión latina y notas por Miguel Antonio Caro. Publicadas por J. M. Rivas Sacconi. - Bogo- tá, Edit. Voluntad, 1947, xxxII241 págs. (Publicaciones del Instituto Caro y Cuervo).

842. Montoto, S. - Cartas inéditas del poeta $D$. Juan de Salinas, con anotaciones de D. Juan de Jáuregui. - BAE, 1946, xxv, núm. 117, p. 137-145.

843. Green, O. H. - Villamediana as correo mayor in the Kingdom of Naples. - HR, 1947, xv, 302306.

844. Rodríguez-Moñino, A. - El poeta Don Gómez de la Rocha (1652-1687). - RCEE, 1945, I, 7-44.

\section{Portugal}

845. Camões, Luis de - Obras completas. Vol. II. Pref. de H. Cidade. - Lisboa, Livraria Sá da Costa, 1946, 309 págs. (Clássicos Sá da Costa).

846. Camôes, Luis VAZ DE - Los Lusíadas. Trad. de M: Aranda y San Juan.-Buenos Aires, Emecé, 1946, 364 págs.

847. Bittencourt, Pedro Calmon MonIz DE - O estado e o direito n'os Lusiadas. - Rio de Janeiro, Dois Mundos, [1945], 224 págs.

848. Almeida, Francisco Filinto DE - Camoneana. - Rio de Janeiro, Jornal do Comércio, 1945, 8 págs., ilustr.

849. Bowra, C. M. - From Virgil to Milton. - New York, 1945. [So. bre Camoes].

850. González-Blanco, P. - Vida $y$ tribulaciones de Luis de Camoens. - México, Botas, 1946, 356 págs.

\section{Autores modernos}

\section{España}

851. Cano, J. L. - Revistas españolas de poesía; 1939-1946. Insula, 1946, 1, núm. 11.

852. Turnbull, Eleanor Laurelle - Contemporary Spanish poetry. Selections from ten poets, translated by Eleanor L. Turnbull, with 
Spanish originals and personal reminiscences of the poets by Pedro Salinas. - Baltimore, The Johns Hopkins Press, 1945, xIII-401 págs., $\$ 3.50$.

853. Guillén, Jorge - Jardines españoles: Antonio Machado, Pedro Salinas, Dámaso Alonso y García Lorca. - UNC, 1946, núm. 6, 153-165.

854. Díaz, J. S. - Diccionario general de bibliografía española. $-\mathrm{BH}$, 1947, vir, núm. 1, 35-44.

855. Adams, N. B. - A note on Mme. Cottin and the Duke of Rivas. - HR, 1947, xv, 218-221.

856. Pattison, W. T. - On Espronceda's personality. - PMLA, 1946, LXI, 1126-1145.

857. Leslie, J. K. - Towards the vindication of Zorrilla: The $\mathrm{Du}$ mas-Zorrilla question again.--HR, 1945, xill, 288-293.

858. Gullón, R. - Tassara, duque de Europa. - BBMP, 1946, xxII, 132-169.

859. BÉCquer - Las rimas y otras páginas. Pról. y notas de José $\mathrm{Ma}$ ría Monner Sans. - Buenos Aires, Ángel Estrada y Cía., 1947, 596 págs. (Colección Clásicos Castellanos).

860. Monner Sans, J. M. - Las fuentes de las "Rimas" becquerianas. - BAAL, 1946, xv, 447-474.

861. Monner Sans, J. M. - Notas sobre Gustavo Adolfo Bécquer. BAAL, 1946, xv, 273-286.

862. Monner Sans, J. M. - Algo sobre Bécquer, poeta y prosista. A, 1947, Lxxxvi, 123-136.

863. Alonso Cortés, N. - Una nota sobre Bécquer. - BSS, 1947, XXIV, 155-156.

864. Fernández Almagro, M. La poesía de Unamuno. - Insula, 1947, II, núm. 14.

865. Machado, A. - Poesie. A cura di O. Macrí. Testo orig. e trad. a fronte. - Milano, Il Balcone, 1947, 230 págs., L. 260.

866. Bellé, O. - Ser y tiempo en la poética de Antonio Machado. - Buenos Aires, Ed. del autor, 1945.

867. Clavería, C. - Notas sobre la poética de Antonio Machado. HispW, 1945, xxvIr, 166-183; A, 1945, LXxxI, 137-164.

868. Mélich Orsini, J. - En torno al pensamiento de Antonio Machado. - RNC, 1946, vII, núm. 58, 67-87.

869. PREDMORE, R. L. - La visión de Castilla en la obra de Antonio Machado. - HispW, 1946, xxix, 500-506.

870. Jiménez, Juan Ramón - Poesía. (En verso). (1917-1923). Buenos Aires, Losada, 1946, 95 págs., $\$ 1.50 \mathrm{~m} / \mathrm{arg}$.

871. Jiménez, J. R. - Poesie. Trad. con testo a fronte e introd. di F. Tentori.-Modena, Guanda, 1946, 150 págs.

872. Pemán, J. M. - Don Eduardo Marquina. - BAE, 1946, xxv. núm. 119, p. 337-347.

873. Díez-Canedo, Enrique - Epigramas americanos.-México, Joa. quín Mortiz, 1945, 79 págs.

874. Casalduero, Joaquín - Cántico, fe de vida. III. Primera lectura. - Asom, 1946, iI, núm. 3, 5664. [Sobre J. Guillén, Cántico].

875. Casalduero, J.-El movimiento rítmico en el segundo Cántico de Jorge Guillén.-Asom, 1946, II, núm. 1, 14-23.

876. Morby, E. S. - García Lorca in Sweden.-HR, 1946, xIv, 38-46.

877. Alberti, Rafael - Selected poems. Trad. de L. Mallan. New York, New Directions, 1945, 32 págs. sin numerar, $\$ 1.00$.

878. CharRy LARA, F. - Vicente Aleixandre y la moderna expresión lírica. - UNC, 1946, núm. 7. 155-164.

879. LARREA, J. - Ingreso a una transfiguración. - CuA, 1946, v, núm. 5, 289-299. [Sobre Emilio Prados, Jardín cerrado]. 
Portugal

880. Cámara, João de Brito - $O$ modernismo em Portugal. - Funchal, 1944, 68 págs.

881. Pimpão, Álvaro J. da Costa - O romantismo das "Viagens" de Almeida Garrett. - Ocid, 1947, xxxi, 189-204.

882. Quental, Antero de - Testamento filosófico de Antero de Quental. (Antología). Pref. e anotações por Sant'Anna Dionisio. Lisboa, Seara Nova, 1946, 208 págs.

883. Almeida, Antonio Ramos de - Antero de Quental: infância e juventude. - Pôrto, Latina, 1943, 2 vols.

884. Lopes, Francisco Fernandes - Do germanismo em Antero. Atlântico, 1943, núm. 4, p. 43-50.

885. Carvalho, Joaquim de - Sôbre a origem da concepção da inconsciência de Deus em Antero de Quental. - Biblos, 1944, xx, 131141.

886. Carvalho, Ruy Galvão de Carlos de Mesquita $e$ as origens do simbolismo português. [18761916]. - Ocid, 1947, xxxi, 141146.

\section{TEATRO}

\section{Teatro antiguo}

España

887. Cirot, G. - Pour combler les lacunes de l'histoire du drame religieux en Espagne avant Gómez Manrique. - BHi, 1943, xLv, 55-62.

888. Hamilton, T. E. - Spoken letters in the comedias of Alarcón, Tirso and Lope. - PMLA, 1947, LXII, 62-75.

889. González Palencia, Á.-Quevedo, Tirso y las comedias ante la Junta de Reformación. - BAE, 1946, xxv, núm. 117, 43-84.

890. Cirot, G. - A propos d'Encina: coup d'oeil sur notre vieux drame religieux. - $\mathrm{BH}$, 1941, xLIII, 123-151.
891. Torres Naharro, Bartolomé DE - Propalladia, and other works. Edited by J. E. Gillet. - Bryn Mawr, Pa., George Banta Publishing Co., 1943-1946, 2 vols.

892. Cervantes - El cerco de $\mathrm{Nu}$ mancia. Tragedia en cuatro jornadas. Ed. anot. por J. Givanel Mas. - Barcelona, 1945, xL-760 págs. (Publicaciones Cervantinas, Segunda serie, vi).

893. Anibal, C. E. - Sobre: Lope de Vega, El sembrar en buena tierra. Ed. W. L. Fichter. - RRQ, 1946, xxxVII, 252-268.

894. Fichter, W. L. - Sobre: J. de Entrambasaguas, Estudios sobre Lope de Vega. - HR, 1947, xv, 239-243.

895. Morley, S. G. \& C. Bruerton - Addenda to the chronology of Lope de Vega's comedias. - HR, 1947, xv, 49-71.

896. Torre, G. DE-Prisma de Lope de Vega: Lo clásico, lo español, lo universal. CuA, 1947, xxxI, núm. 1, 179-190.

897. Lincoln, J. N. - Saint Ursula, the Infanta Isabel, and Lope de Vega. - Ann Arbor, Michigan, University of Michigan Press, 1947, 17 págs., \$0.50. (Contributions in Modern Philology, núm. 7).

898. Pons, Joseph-S. - L'“art nouveau" de Lope de Vega. - BHi, 1945, xLVII, 71-78.

899. Cirot, G. - "L'allégorie des tireurs à l'arc."-BHi, 1945, xLIV, 171-174 [Sobre Lope de Vega].

900. Fichter, W. L. - The date of Lope de Vega's Santiago el Verde. - HR, 1945, xIII, 243-244.

901. Montoto, S. - Un auto de Lope de Vega rechazado. - BAE, 1946, xxv, núm. 119, 429-433.

902. Saunal, D. - Autour des sources de "Pobreza no es vileza" de Lope de Vega]. - BHi, 1946, XIVIII, 238-246.

903. Torre, G. DE - Nueva interpretación de Lope de Vega. RAmér, 1946, viIr, núm. 24, 328333. 
904. Kohler, E. - A propos de la date de composition de "El ejemplo de casadas" de Lope. - BHi, 1946, XLVIII, 264-269.

905. Wagner, C. P. - The date of Peribáñez.-HR, 1947, xv, 72-83. 906. Alarcón - El tejedor de Segovia. Trad. rusa por F. V. Kelena. - Moscow-Leningrad, publicación del gobierno, 1946, 170 págs., 6 rublos.

907. Calderón de la Barca, PeDRo - Deux Comédies: La vie est un songe; Le médecin de son honneur. Trad. de A. Arnoux. - Paris, Grasset, 1945.

908. Bergamín, J. - Por debajo del sueño. (Calderón, calderoniano).RNC, 1946, vII, núm. 56, 3-18.

909. Leavitit, S. E. - A rare edition of plays attributed to Calderón. - HR, 1947, xv, 216-218.

910. Moreto Cabaña, A. - El desdén con el desdén. Trad. rusa por T. L. Schepkenoy-Koupernek. Moscow-Leningrad, publicación del gobierno, 1946, 151 págs., 6 rublos.

\section{Portugal}

911. Vicente, GiL - Obras completas. - São Paulo, Ed. Cultura, 1946, 2 vols., Cr.120\$00. (Série Clássica Brasileiro-Portuguesa, Os Mestres da Língua).

912. Vicente, Gil - Auto de moralidade da embarcação do Inferno. Textos das duas primeiras Edições avulsas e das Copilações, estudadas por P. Quintela, com um apêndice que contém a Tragicomedia alegorica del Parayso y del Infierno. - Coimbra, 1946, cxv-341 págs., ilustr. (Col. Atlántida).

913. Zeitlin, M. A. - Gil Vicente e o teatro português. - MLForum, 1945, XxIx, 85-87.

914. David, Pierre-Notes sur deux. motifs introduits par Gil Vicente dans l'“Auto da embarção da Glória". - BEPI, 1945.

\section{Teatro moderno}

España

915. Vega, J. - Don Ramón de la Cruz, el poeta de Madrid. - Madrid, Imp. Sistemas de Control, 1945, 171 págs., 28 ptas.

916. Díaz, J. S. - Don Ramón de la Cruz y las ediciones fraudulentas. - BH, 1946, v, núm. 11, 715-722.

917. Vega, Ventura de LA - El hombre de mundo. - Buenos $\mathrm{Ai}$. res, Comisión Nacional de Cultura, 1945, 141 págs. (Instituto Nacional de Estudios de Teatro, Biblioteca Teatral, tomo IV).

918. Río, Á. DEL - Sobre: L. Kirschenbaum, Enrique Gaspar and the social drama in Spain. - RRQ, 1945, xxxvi, 246-248.

919. Unamuno, Mrguel de - La venda. Drama en un acto y dos cuadros. - RNC, 1946, viır, núm. 59, 7-17.

920. García Lorca, Federico - La casa de Bernarda Alba. Drama de mujeres en los pueblos de España. - Buenos Aires, Losada, 1945, 125 págs., $\$ 1.50 \mathrm{~m} / \mathrm{arg}$.

921. García Lorca, Federico-Mariana Pineda. A cura di A. Baldo, con un saggio di O. Macrí. Modena, Guanda, 1946, 160 págs., L. 143.

\section{Portugal}

922. Dantas, Júlio - Antigona. Peça em 5 actos, inspirada na obra dos poetas trágicos gregos, e, em especial, na "Antigona", de Sófocles. - Lisboa, Liv. Bertrand, [1945], 130 págs.

\section{NOVELISTICA}

\section{Autores antiguos}

923. Salinas, Pedro - El 'héroe' literario y la novela picaresca española: Semántica e historia literaria. -RUBA, 1946, Iv, 75-84.

924. Alcalá, M. - Don Juan Ma- 
nuel y Shakespeare: Una influencia imposible. - FyL, 1945, x, 55-67.

925. Montesino Samperio, J. V. Sobre la cuantificación del estilo literario: Una contribución al estudio de la unidad de autor en " $\mathrm{La}$ Celestina" de Fernando de Rojas. - RNC, 1946, viI, núm. 55, 94115; núm. 56, 63-88.

926. Gilman, S. - El tiempo y el género literario en la "Celestina". - $R F H, 1945$, vII, 147-159.

927. Green, O. H. - The Celestina and the Inquisition. - HR, 1947, $\mathrm{xv}, 211-216$.

928. Morais, Francisco DE - Crônica de Palmeirim de Inglaterra. $2^{2}$ parte, tomo II, do capítulo Lxxvi a cxxx. Texto establecido, anotado e com glossário organizado por Geraldo de Ulhoa Cintra. - São Paulo, Anchieta, 1946, 435 págs., $\mathrm{Cr} 50 \$ 00$. (Bibl. de Clássicos para todos).

929. Cervantes Saavedra, Migued. $\mathrm{DE}$ - The first part of the life and achievements of the renowned Don Quixote de la Mancha. Trad. de P. Motteux. Ilustr. de Salvador Dali. - New York, Random House, 1946, xvinI-587 págs., \$2.00. (The Illustrated Modern Library).

930. Cervantes Saavedra, Miguel. DE - Don Quichotte. Trad. de F. de Miomandre. Pról. de J. Estelrich. - Paris, Stock, 1945, 2 vols. (Collection Hispanique).

931. Montoro, S. - Nueva bibliografía cervantino-sevillana. - $\mathrm{BH}$, 1947, vi, núm. 2, 97-122; núm. 3, 199-225.

932. DaM, C. F. A. VAN - Observaciones al "Quijote" de Rodríguez Marín. - BÄE, 1946, xxv, núm. 118 , p. 299-301.

933. Alonso Cortés, N. - Sobre unas notas del Quijote. - BBMP, 1946, xxII, 170-176.

934. Knowles, E. B., Jr. - Notes on the Madrid, 1605, editions of Don Quijote. - HR, 1946, xIv, 47-58.
935. Cortines y Murube, F. - Un heraldo de Cervantes en el siglo dieciocho. - AUH, 1946, Ix, 99105.

936. Buenaventura, E. - El Quijote de Cervantes y el Quijote de Unamuno. - UNC, 1946, núm. 7, 143-154.

937. Peery, W. - "The cutious impertinent" in "Amends for ladies". - HR, 1946, xIv, 344-353.

938. Pinilia, N. - Cervantes y Baty. - A, 1946, Lxxxv, 86-93.

939. Xavier, A.- "As novelas exemplares" de Cervantes: A sua importancia inovadora e o seu valor como modelos de que derivou o conto moderno. - Por, 1946, núms. 34, 127-142.

940. Frank, Rachel - Deceit in Cervantes' Novelas ejemplares. HR, 1945, XIII, 244-252.

941. García Soriano, J. - Los dos "Don Quijotes". Investigaciones acerca de la génesis de "El Ingenioso Hidalgo" y de quién pudo ser Avellaneda. - Toledo, Imp. Rafael Gómez-Menor, 1944, 292 págs., 35 ptas.

942. Gilman, S. - Alonso Fernández de Avellaneda: a reconsideration and a bibliography. - HR, 1946, xIv, 304-321.

943. Moreno Báez, E. - ¿Hay una tesis en el "Guzmán de Alfarache"? - RUBA, 1945, III, 269. 291.

944. Castillo Solórzano, Alonso DE - Lisardo enamorado. Pról. y notas de E. Juliá y Martínez. Madrid, Real Academia Española, 1947, 331 págs., 22 ptas. (Biblioteca Selecta de Clásicos Españoles, III).

\section{Autores Modernos}

$E s p a \tilde{n} a$

945. Romeu, R. - Les divers aspects de l'humour dans le roman espagnol moderne. - $\mathrm{BHi}$, 1946, XIVIII, 97-146.

946. Peers, E. A. - Enrique Gil y 
Walter Scott. - Insula, 1946, I, núm. 6.

947. Alarcón, Pedro, A. de - Novelas completas. Con un ideario e índice analítico. $2^{\mathrm{a}}$ ed. - Buenos Aires, Librería El Ateneo, 1945, 968 págs., $\$ 9.00 \mathrm{~m} / \mathrm{arg}$.

948. Alarcón, Pedro Antonio de - Lo scandalo. Romanzo. Trad. e introd. di A. Scagnetti. - Roma, Colombo, 1945, xI-359 págs., L. 200.

949. Coe, Ada M. - An unpublish. ed letter from Pérez Galdós. HR, 1946, XIV, 304-342.

950. Kelin, F. - Benito Pérez Galdós entre nosotros. - LitIn, 1945, Iv, núm. 1, 64-66.

951. LízAro, A. - España en su novelista: Galdós. - RevCu, 1945, $\mathrm{xIX}, 42-65$.

952. KRynen, J. - L'esthétisme de Juan Valera.-Salamanca, Universidad de Salamanca, 1946, 97 págs. 27 ptas. (Acta Salmanticensia. Ivssv Senatvs Universitatis Edita. Filosofía y Letras, Tomo II, núm. 2).

953. Revuelta y Revuelta, Luisa - Valera, estilista.-BaCord, 1946, xvir, 25-71.

954. Eorf, S. - Pereda's conception of realism as related to his epoch. - HR, 1946, xIv, 281-303.

955. Rañó de Petracchi, Lilia La condesa de Pardo Bazán: Su vida y su obra. - Buenos Aires, Libr. Hachette, 1946, 166 págs.

956. PosadA, A. - Leopoldo Alas, "Clarín".-Oviedo, Imp. La Cruz, 1946, 239 págs., ilustr. (Publicaciones de la Universidad de Oviedo).

957. Valle InClán, R. DEL - Il tiranno Banderas. Romanzo. Trad. di A. Camerino. - Milano, Bompiani, 1946, 278 págs., L. 200.

958. VAlle IncláN, R. DEL - Memorie del Marchese di Bradomin. A cura di O. Macrí. - Firenze, Sansoni, 1946, xvi-284 págs., L. . 240.

959. Salinas, Pedro - Significación del esperpento o Valle Inclán, hijo pródigo del 98. - CuA, 1947, vi, núm. 2, 218-244.

960. Fein Pastoriza, Delia - El idioma de Valle Inclán en su obra: "Romance de lobos" - BF, 1944, Iv, 85-94.

961. Azonín - La isla sin aurora. Novela. - Barcelona, Edic. Destino, 1944, 156 págs.

962. Azorín-Blanco en azul. Cuentos. - Buenos Aires, Espasa-Calpe, 1944, 152 págs.

963. Azorín - María Fontán. Buenos Aires, Espasa-Calpe Argentina, 1945,150 págs., $\$ 1.50 \mathrm{~m} /$ arg.

964. Azorín - Ante Baroja. - Zaragoza, Librería General, 1946, 284 págs., 15 ptas.

965. Baroja, Pío - Días aciagos. El hotel del cisne. Novela. - Madrid, Biblioteca Nueva, 1946, 296 págs., 30 ptas.

866. Templin, E. H. - Pío Baroja and science. - HR, 1947, xv, 165. 192.

967. Jiménez, J. R. - Platero y yo. A cura di G. M. Bertini. - Milano, Leonardo, 1944, 216 págs., L. 100.

968. Miró, Gabriel - El obispo leproso. Novela. 2 a parte de Nuestro padre San Daniel. Pról. por Gerardo Diego. Ed. conmemorativa emprendida por los "Amigos de Gabriel Miró". - Barcelona, Tip. Altés, 1947, 341 págs. (Obras completas de Gabriel Miró, Vol. xI).

969. Gómez de la Serna, Ramón - Doña Juana la Loca (Seis novelas super-históricas). - Buenos Aires, Clydoc, 1944, 172 págs., $\$ 4.00 \mathrm{~m} /$ arg.

970. Gómez de la Serna, Ramón - El dueño del átomo. - Buenos Aires, Losada, 1945, 228 págs., $\$ 2.50$. $\mathrm{m} / \mathrm{arg}$.

\section{Portugal}

971. Queiroz, José María Eça de - Cartas de Eça de Queiroz. Lisboa, Aviz, 1945, xv-374 págs. 972. Queiroz, José María Eça de 
- Polêmicas de Eça de Queiroz (com as críticas de Pinheiro Chagas, Camilo, Fialho e Bulhão Pato). Pref. e recolha de João Luso. Rio de Janeiro, Dois Mundos, 1945, 348 págs.

973. SÁ, ViCtor DE - Bibliografia Queirosiana. Contendo o pequeno ensaio panorâmico do livro em Portugal. - Braga, Ed. da Biblioteca Móvel, 1945, 76 págs.

974. Lemos, Antero Vieira de Eça de Queiroz, o seu drama e a sua obra. Ensaio crítico. - Pôrto, Latina, [1945], 266 págs., $20 \$ 00$.

975. Segura, Enrique - Vida de Eça de Queiroz. - Madrid, Centro de Estudios Extremeños, 1945, 213 págs., 15 ptas. (Siglo $\mathrm{xx}$ ).

976. Queiroz, Antonio D'Eça de

- Eça de Queiroz. Conferência.Ocid, 1947, xxxi1, 5-20.

977. Bello, José María - Retrato de Eça de Queiroz. - Rio de Janeiro, Agir, 1945, 326 págs.

978. Costa, Francisco - Eça visto por si próprio. - Lisboa, Pereira, 1946, 66 págs., $10 \$ 00$.

979. Simplicio, Roderico - Eça de Queiroz esquizotimico. - Lisboa, 1946, 31 págs.

980. Rodrigues, Felipe. - Eça de Queiroz e a religião. - ILM, 1946, v, núm. 54 .

981. Bernardes, José - Eça de Queiroz. Suas ideas políticas. ILM, 1946, v, núm. 54.

982. Coelho, Jacinto do Prado As ideias $e$ as formas. - Ocid, 1946, 31 págs.

983. Simões, João Gaspar - Freud, Eça de Queiroz e Francisco Costa. - MunL, 11 maio 1946.

984. Ivens, Diogo - Dorian Gray e o padre Amaro [de Eça de Queiroz]. - Ocid, 1947, xxxı, 73-84.

985. Freitas, N. - Eça de Queiroz (En el centenario de su nacimiento). - CuA, 1946, v, núm. 1, 286294.

986. Eça de Queiroz, documentário de uma comemoração. - Recife, Diretoria de Documentação e Cul- tura, Prefeitura Municipal do Recife, 1947, Iv-231 págs.

987. Costa, Francisco - Primavera cinzenta. Romance. - Lisboa, Parceria A. M. Pereira, 1944, 422 págs.

\section{HISTORIA}

\section{España}

988. Romero, J. L. - Sobre la biografía española del siglo xv y los ideales de vida. - CuHEsp, 1944, I y II, 115-138.

989. Carrillo de Huete, Pedro Crónica del Halconero de Juan II Pedro Carrillo de Huete (hasta ahora inédita). Ed. y estudio por J. de Mata Carriazo. - Madrid, Espasa-Calpe, 1946, xv-563 págs., 75 ptas.

990. Mexía, Pedro - Historia del Emperador Carlos V. Ed. y estu. dio por J. de M. Carriazo. - Madrid, Espasa-Calpe, 1945, xcv-619 págs. (Colección de Crónicas Españolas, 7).

991. González de Mendoza, Juan - Historia de las cosas más notables, ritos y costumbres del gran Reino de la China. Ed., probl. y notas de F. García. - Madrid, Aguilar, 1944, LII-396 págs.

992. Quiñones de Benavente, Juan $\mathrm{DE}$ - Corografía breve de la ciudad de Lérida, de sus antigüedades $y$ memorias. Ed. facsímile de la única conocida de esta obra, en Madrid, 1644, por Catalina del Barrio. Nota preliminar de J. Sol Ballespí. - Lérida, Amigos de los Museos, 1946, 42 págs. (Sección de Bibliófilos, Artes Gráficas Ilerda, Vol. vur).

993. Ronríguez-Moñino, A. - Bi. bliografía de Don Vicente Barrantes (1829-1898). - RCEE, 1945, núm. 4, 461-484.

\section{Portugal}

994. Crónica de cinco reis de Portu. gal. Inédito quatrocentista reproduzido do cód. 886 da Biblioteca 
Pública Municipal do Porto; seguido de capítulos inéditos da versão portuguesa da Crónica Geral de Espanha e outros textos. Ed. diplomática e pról. de A. de MagaIhães Basto. - Pôrto, Livraria Civilização, 1946. 420 págs., $50 \$ 00$. (Biblioteca Histórica, Série Régia, Vol. I).

995. Lopes, Fernão - Quadros da Crónica de D. João I. Sel., pref. e notas de Rodrigues Lapa. - Lisboa, Gráfica Lisbonense, 1945, 85 págs.

996. WiCkI, J. - Die "Historia do Malavar" des P. Diogo Gonçalves S. I. Ein Beitrag zur Indologie und Missiologie. - AHSI, 1945, xIv, 73-101.

997. Sousa, Manuel de Faria e Ásia portuguesa. Trad. de Isabel Ferreira do Amaral Pereira de Matos e Maria Vitória Garcia Santos Ferreira. Introd. de M. Lopes d'Almeida. - Pôrto, Livraria Civilização, 1945. (Biblioteca histórica de Portugal e Brasil. Serie ultramarina, vi).

998. Leal, Raúl - Sociologia de Oliveira Martins. - Pôrto, Liv. Figueirinhas, 1945, 284 págs.

999. Ramos, Feliciano - Alberto Sampaio e a subjectividade das suas interpretações históricas. - Biblos, 1945, xxI, 395-416.

\section{LITERATURA RELIGIOSA}

1000. FIdel de Ros, P. - Los místicos del Norte y Fray Luis de Granada. - AIA, 1947, viI, 5-30.

1001. Teresa de Jesús - The complete works of Saint Teresa of Jesus. Trad. y edit. de E. A. Peers. - New York-London, Sheed \& Ward, 1946, 3 vols., \$15.00.

1002. Peers, E. A. - Saint Teresa, foundress. - BSS, 1946, хxıII, 248260

1003. Alonso Contés, N. - Pleitos . de los Cepedas. - BAE, 1946, xxv, núm. 117, 85-110.

1004. RicARD, R. - Quelques re- marques sur les "Moradas" de Sainte Thérèse. - BHi, 1945, xuviI, 187-198.

1005. JUAN DE LOS ÁNGeles. - Diálogos de la conquista del reino de Dios. Pról. y notas de Á. González Palencia. - Madrid, Real Academia Española, 1946, 360 págs. (Biblioteca Selecta de Clásicos Españoles).

\section{TRATADOS, ENSAYOS Y DISCURSOS}

\section{Autores antiguos}

España

1006. Bataillon, M. - Juan de Valdés. - Lu, 1945, vir, 1-60.

1007. Castro, A.-Antonio de Guevara. Un hombre y un estilo del siglo xvi. - BICC, 1945, I, núm. $1,46-67$.

1008. Estudios acerca de Fray Antonio de Guevara en el iv centenario de su muerte. (Mondoñedo, 3 de abril de 1545). - AIA, 1946, año vi, núms. 22-23.

1009. Fellheimer, Jeannette Hellowes' and Fenton's translations of Guevara's Epístolas familiares. - SPh, 1947, xLIV, 140-156.

1010. Sánchez y Escribano, F. La colaboración en la Philosophía vulgar de Juan de Mal Lara. HR, 1947, xv, 308-312.

1011. Eisenschiml, Otтo - The art of wordly wisdom: Three hundred precepts for success based on the original work of Baltasar Gracián. - New York, Duell, Sloan \& Pearce, 1947, 160 págs., \$2.50.

1012. Maldonado de Guevara, F. - El ocaso de los héroes en "El criticón". - Zaragoza, Institución "Fernando el Católico", 1945, 32 págs., 6 ptas. (Archivo de Filología Aragonesa. Anejo).

\section{Portugal}

1013. Ferreira, Tito Lívio $-F i$ losofias de solteirão em Carta de guia de casados $e$ outras Cartas 
familiares. - BSEF, 1945, I, núm. $1,145-152$.

\section{Antores modernos}

1014. Feijóo y Montenegro, BeNITO JeRónImo - Dos discursos de Feijóo sobre América. Introd. y notas de A. Millares Carlo. - México, Secretaría de Educación Pública, 1945, xxI-[23]-75 págs. (Biblioteca Enciclopédica Popular, 40).

1015. Sánchez Agesta, L. - Feijóo y la crisis del pensamiento político español en el siglo xviii. - RevEP, 1945, xII, núms. 22-23, 71-127.

1016. EIJÁN, S. - Ideas literarias del $P$. Feijóo. Discurso de ingreso en la Real Academia Gallega. - BAGallega, 1944, xxIv, núms. 277. 280, 35-50.

1017. RiCARD, R. - Sur quelques citations latines de Feijóo.-BHi, 1946, xuviI, 269-270.

1018. Zaragüeta, J., I. González, S. Mingüijon, \& J. Corts GraU - Balmes, filósofo social, apologista y político. Pról. de C. Viñas y Mey. - Madrid, Consejo Superior de Investigaciones Científicas, 1945, 484 págs., 25 ptas.

1019. Ganivet, Á. Idearium spagnolo. A cura di C. Bo. - Milano, Muggiani, 1946, 178 págs., L. 196.

1020. Ganivet, Á. - Le fatiche dell' infaticabile creatore Pio Cid. Trad. di C. Bo. - Milano, Il Balcone, 1946, 440 págs., L. 300.

1021. Unamuno, Miguel de - La dignidad humana. $2^{\mathrm{a}}$ ed. - Buenos Aires, Espasa-Calpe Argentina, [1945], $\$ 1.50 \mathrm{~m} / \mathrm{arg}$.

1022. Unamuno, Miguel de - Perplexities and paradoxes. Trad. de S. Gross. - New York, Philosophical Library, 1945, 165 págs., \$2.50.

1023. Azorín - Leyendo a los poetas. - Zaragoza, Librería General, [1945], 231 págs., 12 ptas.

1024. Azorín - Memorias inmemoriales. - Madrid, Biblioteca Nueva, 1946, 345 págs., 40 ptas.

1025. Azorín - El artista y el esti. lo. Ensayos coleccionados, ordenados y precedidos de nuevo estudio acerca de la vida y obra de Azorín por A. Cruz Rueda. - Madrid, Aguilar, 1946, 524 págs., 18 ptas.

1026. García BaCca, J. D. - Ortega y Gasset o el poder vitamínico de la filosofía. - UnivNL, 1946, núm. 6, 25-32.

1027. Gómez de la Serna, Ramón - Gollerías. Ilustr. por el autor. Buenos Aires, Losada, 1946, 174 págs., $\$ 2.50 \mathrm{~m} / \mathrm{arg}$.

1028. Moreno Villa, José - Leyendo a San Juan de la Cruz, Garcilaso, Fr. Luis de León, Bécquer, Rubén Dario, Juan Ramón Jiménez, Jorge Guillén, Federico García Lorca, Antonio Machado, Goya, Picasso. - México, El Colegio de México, 1946, 155 págs.

1029. Domenchina, Juan José-Crónicas de "Gerardo Rivera". 2a ed. - México, Ed. Centauro, 1946, 246 págs.

1030. Gaos, J. - Filosofía y literatura según un filósofo español. CuA, 1945, rv, núm. 6, 109-118.

1031. Nicol, Eduardo - La idea del hombre.-México, Edit. Stylo, 1946, 496 págs.

1032. Ros, Antonio - Mientras el cañón retumba... Un español en Egipto. Pról. de Enrique Díez-Canedo. Portada y dibujos de B. García Ascot. - México, Edit. Cultura, 1946, 358 págs.

\section{MEMORIAS, EPISTOLARIOS Y VIAJES}

1033. Brochado, José da Cunha Cartas. Sel., pref. e notas de António Álvaro Dória. - Lisboa, Sá da Costa, 1944, Lxxiv-163 págs.

\section{PERIODISMO}

1034. Simón Díaz, J. - El Artista. (Madrid, 1835-1836). — Madrid, Consejo Superior de Investigaciones Científicas, 1946, 167 págs. (Colección de Indices de Publicaciones Periódicas). 


\section{FOLKLORE}

1035. Sande, Morś́s M. DE - Del folklore garrovillano: usos y costumbres. - RCEE, 1945, núm. 4, 447 460.

1036. Ricard, R.--Les fêtes de "Moros y cristianos" à Juviles (Prov. de Grenade). - BHi, 1946, xuviII, 263-264.

1037. PÉrez Vidal, J. - La fiesta de S. Juan en Canarias. Ensayo folklórico. - La Laguna de Tenerife, Instituto de Estudios Canarios, 1945, 98 págs., 10 ptas. (Tradiciones populares, vol. III).

1038. Tovar y R., E. D. - Apuntes para una paremiología hispanoamericana. - RJav, 1945, xxIv, 40-45.

1039. Serrano Plaja, Arturo Hijo del alba; villancicos, canciones, ensaladillas y coloquios pastoriles de nochebuena. Sel. y pról. de A. Serrano Plaja. - Buenos Aires, Imp. López, 1943, 103 págs. 1040. Lima, Sílvio Júlio AlbuQUERQUe - Afinidades da poesia popular de Portugal e Galicia. $R B R J$, 1943, III, núm. 6, 122134.

1041. Ṕ́rez Vidal, J.-Influencias geográficas en la poesía tradicional canaria. - Madrid, Aguirre, 1944, 35 págs. (Publicaciones de la Real Sociedad Geográfica, Serie B, núm. 158).

1042. Devoto, D. - Sobre: Cancionero musical español de los siglos $x v$ y xvi, transcripto y comentado por Francisco Asenjo Barbieri. $3^{a}$ ed. $-R F H, 1946$, viII, $167-$ 170.

1043. Devoto, D. - Sobre: Luys de Narváez, Los seys libros del Delphin de música de cifra para tañer vihuela. - RFH, 1946, virI, 171174. 